\title{
Estudo da influência do teor de fibras e de compatibilizante na obtenção de compósitos de polietileno de alta densidade pós-consumo (PEADpc) com fibras de bagaço de cana-de-açúcar (BCA)
}

\author{
Study of the influence of fiber content and compatibilizer in obtaining post-consumer \\ high-density polyethylene (HDPEpc) composites with sugarcane bagasse fibers (BCA)
}

1 Elaine Vidal Dias Gomes Líbano elainevdg@yahoo.com.br

1 Patrícia Soares da Costa Pereira

2 Daniele Cruz Bastos

3 Karolina Vieira de Souza Coelho

\footnotetext{
1 Doutora em Ciência e Tecnologia de Polímeros - Centro Universitário Estadual da Zona Oeste

2 Doutora em Ciências em Engenharia Metalúrgica e de Materiais - Centro Universitário Estadual da Zona Oeste

3 Tecnóloga em Polímeros - Centro Universitário Estadual da Zona Oeste
}

\section{Resumo}

Observa-se uma tendência no mercado de utilizar novos materiais compósitos, obtidos a partir de resíduos sólidos e matriz polimérica, para obter produtos ecologicamente corretos, reduzir o custo de produção, diminuir o desperdício, valorizar a matéria-prima e principalmente buscar uma reutilização para os resíduos da agroindústria. O polietileno de alta densidade (PEAD) é um dos principais resíduos sólidos gerados pelo homem, sendo facilmente encontrado nos aterros sanitários brasileiros. Toneladas de resíduos lignocelulósicos são geradas no país. O PEAD e o bagaço de cana-de-açúcar (BCA) podem ser reaproveitados na produção de materiais compósitos junto com os agentes compatibilizantes que agem na interface, proporcionando uma adesão mais adequada. Neste trabalho, foram preparados compósitos de PEAD pós-consumo (PEADpc), BCA 10, 20 e $30 \%(\mathrm{~m} / \mathrm{m})$ com e sem o uso de agente compatibilizante 3 e $5 \%(\mathrm{~m} / \mathrm{m})$ em extrusora dupla-rosca. Para realização dos ensaios físico-mecânicos, estruturais e térmicos, as amostras foram prensadas. Independente do teor de fibras e da presença do compatibilizante, ocorreu uma redução nos valores de dureza shore D e índice de fluidez, tendência observada em todos os compósitos. Não houve mudança significativa nos valores de massa específica.

\section{Palavras-chave}

Compósitos. PEAD pós-consumo. Compatibilizante. Bagaço de cana-de-açúcar.

\begin{abstract}
There is a tendency in the market to use new composite materials, obtained from solid waste and polymer matrix, to obtain environmentally friendly products, reduce production costs, reduce waste, valorize the raw material and mainly seek reuse for agribusiness waste. High density polyethylene (HDPE) is one of the main man-made solid wastes and is easily found in Brazilian landfills. Tons of lignocellulosic waste are generated in the country. HDPE and sugarcane bagasse fibers (SCB) can be reused in the production of composite materials together with compatibilizing agents that act at the interface, providing a more adequate adhesion. In this work, post-consumption HDPE (HDPECP), SCB 10,20 and $30 \%(\mathrm{~m} / \mathrm{m})$ composites were prepared with and without the use of compatibilizing agent 3 and $5 \%(\mathrm{~m} / \mathrm{m})$ in a twin-screw extruder. To perform the physical-mechanical, structural and thermal tests, the samples were pressed. Regardless of fiber content and compatibilizer presence, there was a reduction in shore $D$ hardness and melt index values, a trend observed in all composites. There was no significant change in specific mass values.
\end{abstract}

\section{Keywords}

Composites. Post-consumption HDPE. Compatibilizer. Sugarcane bagasse.

\section{Como você deve citar?}

LÍBANO, Elaine Vidal Dias Gomes et al. Estudo da influência do teor de fibras e de compatibilizante na obtenção de compósitos de polietileno de alta densidade pós-consumo (PEADpc) com fibras de bagaço de cana-de-açúcar (BCA). Cadernos UniFOA, Volta Redonda, n. 43, p. 49-61, agosto 2020. 
Estudo da influência do teor de fibras e de compatibilizante na obtenção de compósitos de polietileno de alta densidade pósconsumo (PEADpc) com fibras de bagaço de cana-de-açúcar (BCA)

\section{INTRODUÇÃO}

A produção de novos materiais com reutilização de resíduos é uma tendência do mercado que visa ao mesmo tempo obter produtos ecologicamente corretos, reduzir o custo de produção, diminuir o desperdício, valorizar a matéria-prima e principalmente buscar a reutilização desses resíduos. O PEAD é extremamente resistente, levando anos para se degradar na natureza, e ocasiona um grande volume de material a ser descartado em aterro sanitário. Sendo o Brasil um país caracterizado pela economia e com potencialidades de se tornar um dos maiores produtores de fibras vegetais, o desenvolvimento de compósitos reforçados com essas fibras pode resultar em notável crescimento econômico, além de contribuir para o desenvolvimento sustentável. Milhares de toneladas de produtos de resíduos lignocelulósicos são geradas no país. O bagaço pode ser reaproveitado como combustível, gerando energia, como adubo, ração e produção de materiais compósitos (CESTARI et al., 2018; El-FATTAH et al., 2015; SANJAY et al., 2018; VÄISÄNEN et al., 2016; VÄISÄNEN et al., 2017).

Fibras naturais de coco, sisal, bambu, banana, casca de arroz, dentre outras, são materiais ecológicos que têm demonstrado ser um bom reforço para matrizes poliméricas, reduzindo a massa específica e o custo do compósito resultante, o que tem despertado o interesse da indústria (MOKHOTHU ET AL., 2015; MOTAUNG ET AL., 2017; MULINARI ET AL., 2017; OLIVEIRA, 2018).

A natureza apolar (hidrofóbico) do PEAD o torna incompatível com as fibras de bagaço de cana-de-açúcar que, por sua vez, apresentam caráter polar (hidrofílica), dificultando a interação entre fibra e polímero. Assim, torna-se imprescindível o uso de um agente compatibilizante, que poderá reduzir as tensões interfaciais entre a carga e a matriz, proporcionando um maior molhamento, bem como uma adesão mais adequada, facilitando a transferência de esforços entre os componentes (CESTARI et al., 2018; NETO et al., 2014; KU et al., 2011; LIGOWSKI et al., 2015).

O objetivo deste trabalho foi avaliar a influência do teor de fibras de BCA 10, 20 e $30 \%(\mathrm{~m} / \mathrm{m})$ e do compatibilizante (PE-g-MA) 3 e $5 \%(\mathrm{~m} / \mathrm{m})$ nas propriedades físico-mecânicas, estruturais e térmicas dos compósitos, utilizando-se o polietileno de alta densidade pós-consumo (PEADpc).

\section{MATERIAIS E MÉTODOS}

O PEAD pós-consumo foi fornecido pela empresa de Reciclagem Peterlu, Seropédica, Rio de Janeiro. As fibras de bagaço de cana-de-açúcar foram gentilmente cedidas pela HC Sucroquímica, sediada na cidade de Campos dos Goytacazes-RJ, Brasil, sendo utilizado como reforço após a sua moagem e classificação granulométrica. $O$ agente compatibilizante, um polietileno funcionalizado (PEAD enxertado com anidrido maleico - PE-g-MA) foi fornecido pela Polybond, sob o nome comercial de Polybond 3009, com 1,0\% de anidrido maleico (nominal) e índice de fluidez de $4 \mathrm{~g} / 10 \mathrm{~min}$ (ASTM $\left.\mathrm{D} 1238,190^{\circ} \mathrm{C} / 2,16 \mathrm{Kg}\right)$.

\subsection{Obtenção das fibras bagaço de cana-de-açúcar (BCA), moagem e classificação granulométrica}

Inicialmente foi feita a trituração do bagaço com água destilada, na proporção de 3:1. 0 bagaço foi seco a $105^{\circ} \mathrm{C}$, em estufa de aquecimento Marconi (modelo MA 033), por 24 horas, e depois triturado novamente por 10 minutos, a fim de se separar as fibras (CESTARI, 2010). 0 material triturado foi separado no peneirador elétrico Pavitest com peneiras abronzinox $50 \times 50 \times 10 \mathrm{~cm}$ e com telas em inox, a uma velocidade de $80 \mathrm{rpm}$ por 10 minuto. A maior parte do material $(73 \%)$ ficou retido nas peneiras de 50 mesh $(0,297 \mathrm{~mm})$ e 80 mesh $(0,177 \mathrm{~mm})$. Sendo assim, optou-se em utilizar as fibras de tamanho 
menor que 0,297 e maior que $0,177 \mathrm{~mm}$. O beneficiamento do BCA foi realizado no laboratório didático e de pesquisa de polímeros do UEZO.

\subsection{Preparação dos compósitos}

Foram preparados compósitos de PEADpc com BCA $(10,20$ e $30 \% \mathrm{~m} / \mathrm{m})$ e compatibilizante em extrusora dupla-rosca co-rotatória Teck Tril, modelo DCT 20-40, com temperaturas das zonas variando de 70 a $190^{\circ} \mathrm{C}$ da alimentação até a saída da matriz, e rotação de $300 \mathrm{rpm}$. 0 agente compatibilizante utilizado foi o polietileno PE-g-MA ( 3 e $5 \% \mathrm{~m} / \mathrm{m}$ ). 0 material foi, então, picotado em tamanho próximo ao grão de PEADpc original. 0 processamento dos compósitos foi realizado no laboratório didático e de pesquisa de polímeros do UEZO.

\subsection{Obtenção dos corpos-de-prova}

Os corpos-de-prova dos materiais foram confeccionados em prensa hidráulica de aquecimento, marca Marconi, modelo MA 098, a partir da prensagem das amostras peletizadas que foram obtidas durante a extrusão. Sob a temperatura de $190^{\circ} \mathrm{C}$, com 1 minuto na prensa quente para fundir e, depois, durante 3 minutos e com pressão de 5 toneladas para formação do filme, retiradas e, em seguida, foram resfriadas na prensa fria durante 3 minutos, obtendo-se os filmes dos materiais (LíBANO et al., 2019). A Tabela 1 apresenta a formulação dos materiais.

Tabela 1 - Composição dos materiais obtidos.

\begin{tabular}{llll}
\hline Amostras & PEAD $(\% \mathrm{~m} / \mathrm{m})$ & BCA $(\% \mathrm{~m} / \mathrm{m})$ & PE-g-MA $(\% \mathrm{~m} / \mathbf{m})$ \\
\hline PEADpc & 100 & 0 & 0 \\
\hline PEADpc/BCA10 & 90 & 10 & 0 \\
\hline PEADpc/BCA20 & 80 & 20 & 0 \\
\hline PEADpc/BCA28,5/PE-g-MA5 & 66,5 & 28,5 & 5 \\
\hline PEADpc/BCA29,1/PE-g-MA3 & 67,9 & 29,1 & 3 \\
\hline PEADpc/BCA30 & 70 & 30 & 0 \\
\hline
\end{tabular}

Fonte: dos autores, 2020

\subsection{Caracterizações}

\subsubsection{Massa Específica}

A análise de massa específica das amostras foi executada, segundo a norma ASTM D792-13 (2013). O equipamento (modelo DSL 910- GEHAKA) forneceu o valor da massa específica do material analisado. Para cada amostra, foram excluídos o maior e o menor valor, calculando-se a média aritmética das cinco determinações (LíBANO et al., 2019). A análise foi realizada no laboratório didático e de pesquisa de polímeros do UEZO.

\subsubsection{Dureza}

Os ensaios de dureza foram executados, segundo a norma ASTM D2240-05 (2010). O Durômetro Shore D (Type GS 702) forneceu o valor da dureza Shore D do material analisado. Para cada amostra, foram excluídos o maior e o menor valor, calculando-se a média aritmética das cinco determinações (LÍBANO et al., 2019). A análise foi realizada no laboratório didático e de pesquisa de polímeros do UEZO. 
Estudo da influência do teor de fibras e de compatibilizante na obtenção de compósitos de polietileno de alta densidade pósconsumo (PEADpc) com fibras de bagaço de cana-de-açúcar (BCA)

\subsection{3 Índice de fluidez (MFI)}

O índice de fluidez das amostras foi avaliado, segundo a norma ASTM D-1238-01 (2002) no Medidor de índice de fluidez (CEAST- Quick Index). Foram utilizados 5,00g de cada amostra, a uma temperatura de $190^{\circ} \mathrm{C}$ e submetido à carga de $2,160 \mathrm{Kg}$. A partir dos dados obtidos, foi calculado 0 valor do índice de fluidez, cuja unidade é expressa em gramas/10 minutos. Para cada amostra, foram excluídos o maior e o menor valor, calculando-se a média aritmética das cinco determinações (LíBANO et al., 2019). A análise foi realizada no laboratório didático e de pesquisa de polímeros do UEZO.

\subsubsection{Absorção de água}

O ensaio de absorção de água foi realizado, conforme a norma ASTM D570-98 (1999) no laboratório de tecnologia de materiais do UEZO. Foram preparados corpos-de-prova feitos a partir de filmes prensados. As amostras foram pesadas (M1) após secas em estufa, em temperatura de $50^{\circ} \mathrm{C}$ por $24 \mathrm{~h}$ (BECKER et al., 2014). Cada amostra foi mergulhada na água destilada em temperatura ambiente, durante períodos de 24, 72 e 384 horas, tendo seu peso medido (M2) após a secagem manual das amostras. A absorção de água (WA) foi, então, calculada conforme a fórmula:

$$
W A(\%)=\frac{(100 x(M 2-M 1))}{M 1}
$$

\subsubsection{Difratometria de raios $X(D R X)$}

A estrutura cristalina do PEADpc e dos compósitos foi investigada por difratometria de raios $X$ no Difratômetro de raios-X Rigaku Miniflex, modelo DMAX 2200. Os difratogramas foram obtidos utilizando-se tensão de $40 \mathrm{kV}$ e corrente de $30 \mathrm{~mA}$, com ângulo de varredura entre 2-50', usando-se a amostra dos compósitos e PEADpc sob forma de filme de $1 \mathrm{~mm}$ de espessura (CESTARI, 2010). A análise foi realizada no Instituto de Macromoléculas Professora Eloisa Mano - IMA.

\subsubsection{Calorimetria Exploratória Diferencial (DSC)}

A análise de DSC foi feita em equipamento Calorímetro Exploratório Diferencial TA, modelo Q1000, utilizando-se panelinhas de alumínio. As condições de análise foram: taxa de aquecimento de $10^{\circ} \mathrm{C} /$ min; faixa de temperatura variando da temperatura ambiente até $200^{\circ} \mathrm{C}$. Através da técnica de DSC foram avaliadas a temperatura de fusão cristalina $\left(T_{m}\right)$, a temperatura de cristalização ocorrida durante o resfriamento $\left(T_{c c}\right)$ e o grau de cristalinidade $\left(X_{c}\right)$ de cada amostra, considerando-se as curvas do segundo aquecimento. $O$ grau de cristalinidade $\left(X_{c}\right)$ de cada amostra foi calculado como a razão entre a entalpia de fusão da amostra e a entalpia de fusão do PE $100 \%$ cristalino $\left(\Delta \mathrm{H}^{\mathrm{o}}=293 \mathrm{~J} / \mathrm{g}\right)($ LíBANO et al., 2019). A análise foi realizada no Instituto de Macromoléculas Professora Eloisa Mano - IMA.

\subsubsection{Análise termogravimétrica (TGA)}

A análise termogravimétrica foi realizada no analisador termogravimétrico TA INSTRUMENT Q600, sob atmosfera de Nitrogênio, em uma vazão de $50 \mathrm{~mL} /$ minuto, a uma taxa de aquecimento de $10^{\circ} \mathrm{C} / \mathrm{min}$, temperatura entre 30 e $700{ }^{\circ} \mathrm{C}$ (CESTARI, 2010). A análise foi realizada no Instituto de Macromoléculas Professora Eloisa Mano - IMA. Taxa $50 \mathrm{~mL} / \mathrm{min}$. 


\section{$3 \quad$ RESULTADOS E DISCUSSÃO}

\subsection{Massa específica, Dureza e MFI}

Os valores de massa específica, dureza e MFI de todos os materiais estudados são apresentados na Tabela 2.

Tabela 2 - Influência da adição de BCA e PEAD-g-MA nos ensaios físico-mecânicos de compósitos de matriz PEADpc.

\begin{tabular}{llll}
\hline Amostras & Massa específica $\left(\mathbf{g} / \mathbf{c m}^{3}\right)$ & Dureza (shore $\mathbf{D})$ & MFI (g/10 $\mathbf{~ m i m})$ \\
\hline PEADpc & $1,02 \pm 0,14$ & $79,00 \pm 1,41$ & $0,76 \pm 0,11$ \\
\hline PEADpc/BCA10 & $1,00 \pm 0,04$ & $73,61 \pm 2,16$ & $0,69 \pm 0,06$ \\
\hline PEADpc/BCA20 & $1,04 \pm 0,03$ & $73,80 \pm 1,30$ & $0,55 \pm 0,03$ \\
\hline PEADpc/BCA28,5/PE-g-MA5 & $1,05 \pm 0,11$ & $71,60 \pm 2,79$ & $0,42 \pm 0,03$ \\
\hline PEADpc/BCA29,1/PE-g-MA3 & $1,13 \pm 0,19$ & $70,41 \pm 3,78$ & $0,31 \pm 0,05$ \\
\hline PEADpc/BCA30 & $1,00 \pm 0,03$ & $72,21 \pm 1,81$ & $0,38 \pm 0,03$ \\
\hline
\end{tabular}

Fonte: dos autores, 2020

Não foram observadas mudanças nos valores de massa específica dos compósitos em relação ao PEADpc, pois apresentaram uma massa específica praticamente inalterada independente do teor de fibra ou da presença do compatibilizante utilizados. Entretanto, houve um aumento de $10 \%$ no valor da densidade do compósito com menor teor de compatibilizante (PEADpc/BC29,1/PA-g-MA3), quando comparado ao polímero puro que pode ter ocorrido pelo fato de que o compatibilizante melhorou a adesão da fibra/polímero, reduzindo a presença de espaços vazios na interface e, por consequência, diminuindo, assim, a higroscopicidade da fibra (LEÃO, 2013).

Observou-se uma diminuição da dureza dos compósitos em relação ao PEADpc independente do teor de fibras e da presença do compatibilizante. Segundo Borsoi (2011), esse efeito de redução ocorre devido à provável falta de uniformidade na distribuição das fibras. De acordo com Jesus (2014), fibras naturais podem promover uma redução na resistência mecânica à penetração, por consequência da higroscopicidade das fibras, portanto, conforme aumenta o seu conteúdo, aumenta o teor de umidade do material. As moléculas de água teriam um efeito plastificante na superfície e, como o teste de dureza é feito na superfície, as fibras podem proporcionar também maior absorção de umidade e, consequentemente, uma queda na dureza (JESUS, 2014).

Assim como na análise de dureza, observou-se a diminuição no índice de fluidez dos compósitos independente do teor de fibras e da presença do compatibilizante. Nos compósitos com maiores teores de fibra (PEADpc/BCA30 e PEADpc/BCA29,1/PE-g-MA3), essa redução foi mais significativa, visto que o reforço impede a mobilidade da cadeia polimérica (Bedin, 2014). Nos compósitos com adição de PEAD-g-MA, observou-se uma redução de, aproximadamente, 40 e $55 \%$ para os teores de 5 e $3 \%$ de compatibilizante, respectivamente. 0 compatibilizante melhorou a interação das fibras com a matriz, impedindo mais a movimentação da cadeia polimérica, fazendo com que o índice de fluidez diminuísse significantemente. O MFI é um parâmetro definido experimentalmente, inversamente proporcional à viscosidade do material fundido a uma temperatura e taxa de cisalhamento especificadas pelas condições operacionais de medida. A adição da carga de reforço normalmente diminui o MFI dos materiais compósitos, pois a presença das fibras na matriz polimérica reduz a mobilidade das cadeias, restringindo o fluxo do polímero fundido e aumentando, portanto, a viscosidade (JESUS, 2014; BASTOS et al., 2018). 
Estudo da influência do teor de fibras e de compatibilizante na obtenção de compósitos de polietileno de alta densidade pósconsumo (PEADpc) com fibras de bagaço de cana-de-açúcar (BCA)

\subsection{Absorção de água}

A absorção de água é um parâmetro importante na obtenção de compósitos com fibras naturais, pois a alta higroscopicidade reduz as propriedades mecânicas do material e promove alterações das dimensões (BECKER et al., 2014; SPINACÉ et al., 2009). A quantidade de água absorvida pelo PEADpc e os compósitos com BCA e o PE-g-MA é apresentada na Figura 1. A variação entre as medidas foi desprezível, não sendo possível visualizar a barra de erros no gráfico da Figura 1.

Figura 1 - Absorção de água do PEADpc e de seus compósitos com a variação do tempo de 24, 72 e 384 horas.

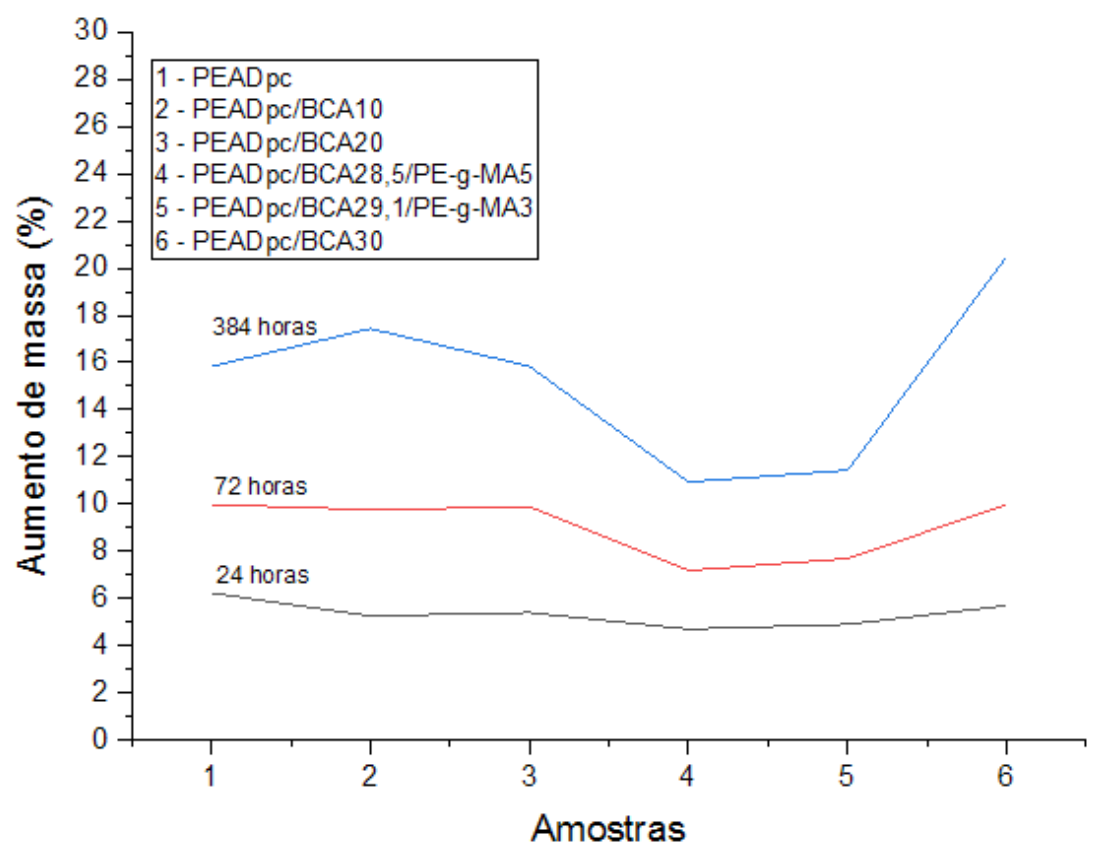

Fonte: dos autores, 2020

Segundo Kaymakci et al. (2016), a absorção de água nos compósitos termoplásticos pode ocorrer através de poros finos, espaços entre o polímero e o material de enchimento e microfissuras na matriz polimérica. 0 efeito esperado por essa análise era de que a adição das fibras de BCA aumentasse a taxa de absorção de água, devido à presença de grupos hidroxilas na celulose da fibra natural, propiciando uma característica hidrofílica à matriz hidrofóbica do PEADpc. Considerando os compósitos sem compatibilizante, os resultados mostraram que, em tempos menores (24 e 72 horas), a presença da fibra não promoveu alteração na quantidade de água absorvida dentro de um mesmo intervalo de tempo. À medida que aumenta o tempo de absorção (384 horas), de uma maneira em geral, há um aumento de massa dos compósitos.

Nos compósitos com compatibilizante (Amostra 4: PEADpc/BCA28,5/PE-g-MA5 e Amostra 5: PEADpc/BCA29,1/PE-g-MA3), em tempos maiores (72 e 384 horas), a presença do PE-g-MA promoveu uma redução da massa. 0 compatibilizante atua promovendo ligações covalentes ou outras interações entre fibra e matriz, reduzindo a absorção de água nesses sistemas. Ao considerar que as moléculas de água são acumuladas nas fibras altamente hidrofílicas presentes nesses sistemas, uma efetiva ligação ou interação das fibras com a matriz ou com os agentes de acoplamento é capaz de reduzir a absorção de água dos compósitos (SANTOS, 2007). 


\subsection{Difratometria de raios X (DRX)}

A Figura 2 apresenta os difratogramas de DRX do PEADpc e dos compósitos PEADpc/BCA sem e com compatibilizante. O PEADpc apresenta picos de intensidade bem definidos que mostraram a existência da fase cristalina com os ângulos $2 \theta$ localizados próximos a 22 e $24^{\circ}$ e que são característicos dos planos (110) e (200) (CESTARI, 2010). Os difratogramas dos compósitos, independentes do teor de fibras e da presença de compatibilizante, apresentaram os mesmos picos que o PEADpc, porém com um discreto deslocamento do pico para ângulos menores e com menor intensidade. Isso ocorreu possivelmente devido ao material ser reciclado e ter outros componentes em sua composição.

Foi calculado o índice de cristalinidade pelo método da área (Tabela 3), onde a cristalinidade foi expressa pela razão entre a área total das regiões cristalinas e a área total do material (CAO et al., 2008; LEÃO, 2013; TANOBE, 2003;). Apesar dos compósitos apresentarem os perfis das curvas de DRX semelhantes aos do PEADpc, ocorreu uma redução na cristalinidade, tanto pelo aumento do teor de fibras quanto pela presença do compatibilizante. 0 compósito com $30 \%$ de BCA apresentou uma redução de $30 \%$ na cristalinidade. Essa redução, provavelmente, se deve ao fato de que as fibras provocam uma desorganização do empacotamento das cadeias poliméricas, devido à falta de tratamento da fibra (KABIR et al., 2013; PRACELLA et al., 2006).

Figura 2 - Difratogramas de raios $\mathrm{X}$ do PEADpc e dos compósitos com BCA e PE-g-MA.

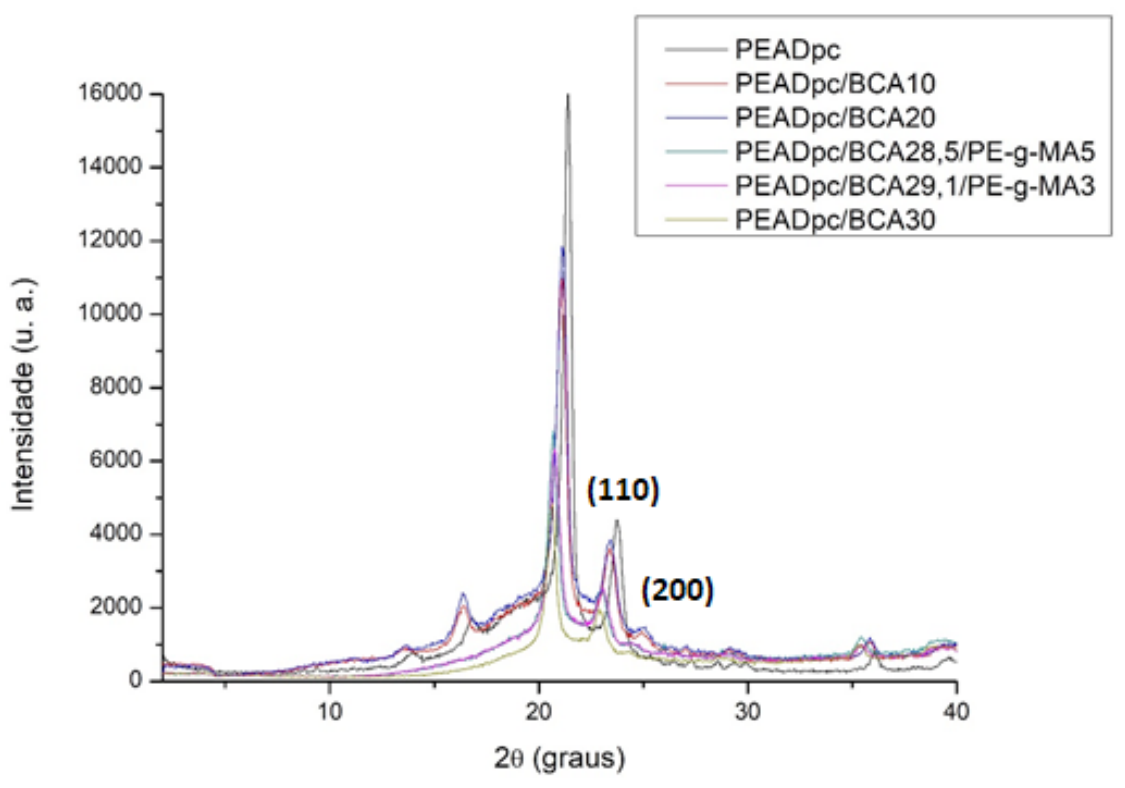

Fonte: dos autores, 2020 
Estudo da influência do teor de fibras e de compatibilizante na obtenção de compósitos de polietileno de alta densidade pósconsumo (PEADpc) com fibras de bagaço de cana-de-açúcar (BCA)

Tabela 3 - Influência da adição de BCA e PEAD-g-MA na cristalinidade dos compósitos de matriz PEADpc.

\begin{tabular}{ll}
\hline Amostras & Cristalinidade (\%) \\
\hline PEADpc & 46,72 \\
\hline PEADpc/BCA10 & 35,22 \\
\hline PEADpc/BCA20 & 33,57 \\
\hline PEADpc/BCA28,5/PE-g-MA5 & 41,67 \\
\hline PEADpc/BCA29,1/PE-g-MA3 & 35,84 \\
\hline PEADpc/BCA30 & 32,66 \\
\hline
\end{tabular}

Fonte: dos autores, 2020

Considerando a presença do compatibilizante, o compósito com maior teor de compatibilizante (5\%) apresentou uma redução de apenas $10 \%$. Uma possível explicação para esse fato é que os compósitos compatibilizados apresentam maior interação interfacial, devido às reações entre os grupos ácidos do anidrido maleico e dos grupos hidrofílicos, presentes na superfície da fibra (ARAÚJO et al., 2008; BORSOl et al., 2011; BORSOI et al., 2014).

\subsection{Calorimetria Exploratória Diferencial (DSC)}

A Tabela 4 apresenta os dados de temperatura de fusão cristalina $\left(T_{m}\right)$, da temperatura de cristalização $\left(T_{c}\right)$ e do grau de cristalinidade $\left(X_{c}\right)$, considerando-se o segundo aquecimento, obtidos por DSC para o PEADpc e para os compósitos com BCA na ausência e presença de PE- $g$-MA. No segundo aquecimento, não foi observada nenhuma variação na linha de base para o BCA, o que significa que o BCA não tem qualquer transição na faixa de temperatura avaliada.

Tabela 4 - Influência da adição de BCA e PEAD-g-MA nas propriedades térmicas dos compósitos de matriz PEADpc.

\begin{tabular}{llll}
\hline Amostras & $\mathbf{T}_{m}\left({ }^{\circ} \mathbf{C}\right)$ & $\mathbf{T}_{c}\left({ }^{\circ} \mathbf{C}\right)$ & $\mathbf{X}_{c}\left({ }^{\circ} \mathbf{C}\right)$ \\
\hline PEADpc & 124,42 & 114,32 & 36,6 \\
\hline PEADpc/BCA10 & 124,32 & 114,35 & 35,6 \\
\hline PEADpc/BCA20 & 124,14 & 114,33 & 33,1 \\
\hline PEADpc/BCA28,5/PE-g-MA5 & 124,31 & 114,13 & 32,3 \\
\hline PEADpc/BCA29,1/PE-g-MA3 & 124,26 & 114,43 & 31,4 \\
\hline PEADpc/BCA30 & 124,36 & 114,81 & 35,2 \\
\hline
\end{tabular}

Fonte: dos autores, 2020

Não houve variação na temperatura de fusão cristalina do PEADpc $\left(124^{\circ} \mathrm{C}\right)$ nos materiais estudados, mostrando que o tamanho médio de seus cristais não sofreu modificação (CESTARI, 2010). Como não houve mudança no valor da $T_{c}\left(114^{\circ} \mathrm{C}\right)$, pode-se inferir que houve pouca influência das partículas de BCA na cristalização do PEADpc. Foi observada uma tendência de diminuição no grau de cristalinidade $\left(X_{c}\right)$ do PEADpc, independente do teor de BCA e da ausência ou presença de compatibilizante, corroborando com os dados do DRX (KABIR et al., 2013; PRACELLA et al., 2006). 


\subsection{Análise termogravimétrica (TGA)}

As curvas de TGA do PEADpc e dos compósitos com BCA sem e com compatibilizante estão representados nas Figuras 3 e 4, e na Tabela 5 são mostradas as temperaturas inicial ( $\left.T_{\text {onset }}\right)$ e final ( $\left.T_{\text {final }}\right)$ de degradação do PEADpc e dos materiais compósitos, a $T_{\text {máx }}$ (temperatura na qual aconteceu o máximo da degradação), e o teor de restante.

Figura 3 - Curvas de TGA para o PEADpc e compósitos sem e com PE-g-MA.

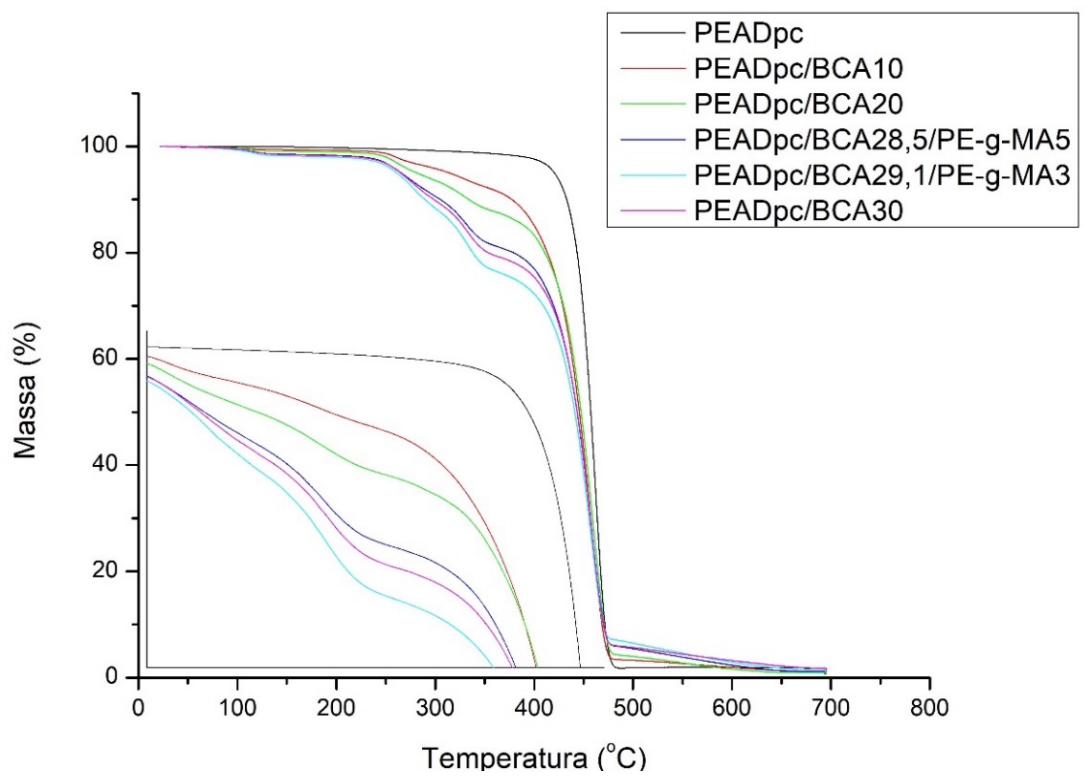

Fonte: dos autores, 2020

Figura 4 - Curvas de DTGA para o PEADpc e compósitos sem e com PE-g-MA.

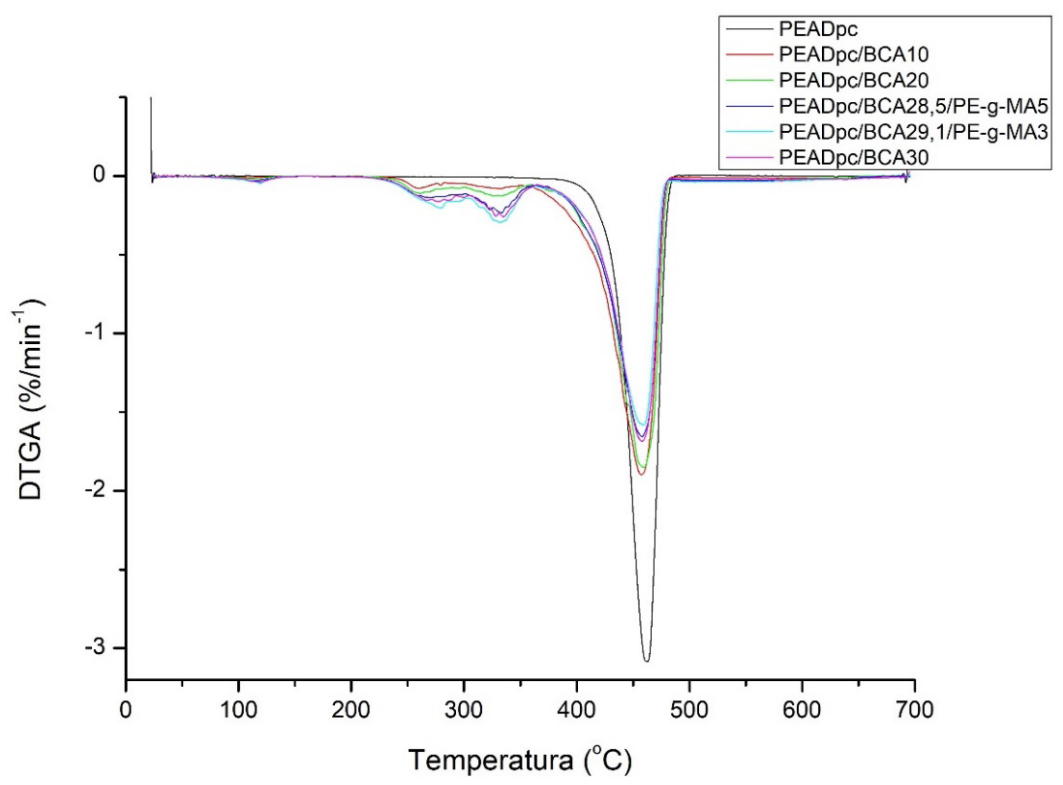

Fonte: dos autores, 2020 
Estudo da influência do teor de fibras e de compatibilizante na obtenção de compósitos de polietileno de alta densidade pósconsumo (PEADpc) com fibras de bagaço de cana-de-açúcar (BCA)

Para o PEADpc, a degradação teve início em $441^{\circ} \mathrm{C}$ e término em $475^{\circ} \mathrm{C}$ (Tabela 5). A adição de BCA em PEADpc promoveu uma diminuição na resistência térmica do material. $A \mathrm{~T}_{\text {onset }}$ e a $\mathrm{T}_{\text {máx }}$ dos compósitos apresentaram 3 etapas distintas que evidenciam as etapas de queima dos componentes celulósicos (hemicelulose, celulose e lignina) e do PEADpc (BARNETO et al., 2010; TEODORO et al., 2011). $\mathrm{A} \mathrm{T}_{\text {final }}$ e o teor de resíduo nos compósitos foram praticamente o mesmo.

Tabela 5 - Influência da adição de BCA e PEAD-g-MA na $T_{\text {onset' }} T_{\text {final }}$ e $T_{\text {máx }}$ do PEADpc e seus compósitos.

\begin{tabular}{lllll}
\hline Amostras & $\mathbf{T}_{\text {onset }}\left({ }^{\circ} \mathrm{C}\right)$ & $\mathbf{T}_{\text {final }}\left({ }^{\circ} \mathbf{C}\right)$ & $\mathbf{T}_{\text {máx }}\left({ }^{\circ} \mathbf{C}\right)$ & Resíduo (\%) \\
\hline PEADpc & 441 & 475 & 462 & 1,77 \\
\hline PEADpc/BCA10 & $245 / 312 / 426$ & 473 & $260 / 332 / 458$ & 1,13 \\
\hline PEADpc/BCA20 & $244 / 314 / 430$ & 477 & $262 / 332 / 460$ & 0,94 \\
\hline PEADpc/BCA28,5/PE-g-MA5 & $246 / 319 / 430$ & 470 & $276 / 335 / 458$ & 1,67 \\
\hline PEADpc/BCA29,1/PE-g-MA3 & $250 / 318 / 429$ & 470 & $279 / 332 / 459$ & 1,34 \\
\hline PEADpc/BCA30 & $242 / 318 / 428$ & 472 & $270 / 333 / 457$ & 1,14 \\
\hline
\end{tabular}

Fonte: dos autores, 2020

\section{CONCLUSÕES}

Compósitos de PEAD pós-consumo (PEADpc) com bagaço de fibra de cana-de-açúcar BCA 10, 20 e $30 \%(\mathrm{~m} / \mathrm{m})$ foram preparados, em extrusora dupla-rosca, com e sem o uso de agente compatibilizante 3 e $5 \%(\mathrm{~m} / \mathrm{m})$. Os resultados obtidos mostraram que não foram observadas mudanças nos valores de densidade dos compósitos em relação ao PEADpc, já que apresentaram uma densidade praticamente inalterada independente do teor de fibra ou da presença do compatibilizante utilizados. Além disso, as fibras de BCA promoveram uma redução na dureza por consequência da higroscopicidade das fibras. A adição da carga de reforço diminuiu o MFI dos materiais compósitos, aumentando, portanto, a viscosidade. A presença do compatibilizante melhorou a interação das fibras com a matriz, o que foi confirmado pela redução do MFI.

Em relação à absorção de água, à medida que aumenta o tempo de absorção, houve um aumento de massa dos compósitos independente do teor de BCA e da presença de compatibilizante. 0 compatibilizante promoveu uma maior interação das fibras com a matriz, diminuindo assim a higroscopicidade da fibra.

Apesar dos compósitos apresentarem os perfis das curvas de DRX semelhantes aos do PEADpc, ocorreu uma redução na cristalinidade, tanto pelo aumento do teor de fibras quanto pela presença do compatibilizante.

Ressalta-se ainda que foi observada uma tendência de diminuição no grau de cristalinidade $\left(X_{c}\right)$ dos compósitos em relação ao PEADpc, independente do teor de BCA e da presença de compatibilizante. A adição de BCA em PEADpc promoveu uma diminuição na resistência térmica do material.

Portanto, conclui-se que as variações nas propriedades físico-mecânicas, estruturais e térmicas dos PEADpc/BCA/ PE-g-MA foram pouco significativas independente do teor de fibras e da presença do compatibilizante, constituindo uma opção para a redução de custos em aplicações industriais e também ajudando na preservação do meio ambiente. 


\section{AGRADECIMENTOS}

Os autores agradecem à Fundação de Amparo à Pesquisa do Estado do Rio de Janeiro (Faperj), à Peterlu e à Polybond.

\section{REFRÊNCIAS}

ARAUJO, J. R.; WALDMAN, W. R. \& DE PAOLI, M. A. Thermal properties of high density polyethylene composites with natural fibres: Coupling agent effect. Polymer Degradation and Stability, v. 93, p.1770-1775,2008.

ASTM D792-13. AMERICAN SOCIETY FOR TESTING AND MATERIALS. Standard Test Methods for Density and Specific Gravity (Relative Density) of Plastics by Displacement. In: Annual Book of ASTM, 2013.

ASTM D2240-05. AMERICAN SOCIETY FOR TESTING AND MATERIALS. Standard Test Method for Rubber Property-Durometer Hardness. In: Annual Book of ASTM, 2010.

ASTM D1238-01. AMERICAN SOCIETY FOR TESTING AND MATERIALS. Melt flow Rates of Thermoplastics by Extrusion Plastometer. In: Annual Book of ASTM, 2002.

ASTM D570-98. AMERICAN SOCIETY FOR TESTING AND MATERIALS. Standard Test Method for Water Absorption. In: Annual Book of ASTM, 1999.

BARNeto, A. G.; CARMONA, J. A.; FERRER, J. A. C.; BLANCO, M. J. D. Kinetic Study on the Thermal Degradation of a Biomass and its Compost: Composting Effect on Hydrogen Production. Fuel - The Science and Technology of Fuel and Energy, v. 89, p. 462-473, 2010.

BASTOS, D. C.; LEÃO, A. G.; PEREIRA, P. S. C. Characterization of Polypropylene Post-Consume / Banana Fiber Composites, Acta Scientiae et Technicae, v. 6, p. 19-22, 2018.

BECKER, D.; KLEINSCHMIDT, A. C.; BALZER. P. S. Compósitos de PVC rígido e fibras de bananeira: Efeito do tratamento da fibra, Revista Matéria, v. 19, p. 257-265, 2014.

BEDIN, Matheus Guilhen. Compósito com polietileno de baixa densidade e fibra de coco in natura e modificada. 2014. Dissertação (Mestrado em Engenharia e Ciência de Materiais) - Centro de Ciência e Tecnologia, Universidade Estadual do Norte Fluminense Darcy Ribeiro, Campos, 2014.

BORSOI, C.; SCIENZA, L. C.; ZATTERA, A. J.; ANGRIZANI, C. C. Obtenção e caracterização de compósitos utilizando poliestireno como matriz e resíduos de fibras de algodão da indústria têxtil como reforço, Polímeros: Ciência e Tecnologia, v. 21, p. 271-279, 2011.

BORSOI, C.; BERWIG. K. H.; SCIENZAC, L. C; ZOPPAS, B. C. D. A; BRANDALISE, R. N; ZATTERA, A. J. Behavior in simulated soil of recycled expanded polystyrene/waste cotton composites, Materials Research, v. 17, p. 275-283, 2014.

CAO, X.; CHEN, Y.; CHANG, P. R.; STUMBORG, M.; HUNEAULT, M. A. Green Composites Reinforced with Hemp Nanocrystals in Plasticized Starch, Journal of Applied Polymer Science, v. 109, p. 3804-3810, 2008. 
Estudo da influência do teor de fibras e de compatibilizante na obtenção de compósitos de polietileno de alta densidade pósconsumo (PEADpc) com fibras de bagaço de cana-de-açúcar (BCA)

CESTARI, Sibele Piedade. Papel sintético sustentável para embalagem. 2010. Tese (Doutorado em Ciência e Tecnologia de Polímeros) - Instituto de Macromoléculas Professora Eloisa Mano, Universidade Federal do Rio de Janeiro, Rio de Janeiro, 2010.

CESTARI, S. P.; ALBITRES, G. A. V.; MENDES, L. C.; ALTSTÄDT, V.; GABRIEL, J. B.; AVILA, G. C. B.; SILVEIRA, I. S. S. Advanced properties of composites of recycled high-density polyethylene and microfibers of sugarcane bagasse, Journal of Composite Materials, v. 52, p. 867-873, 2018.

EL-FATTAH, A. A.; DEMERDASH, A. G. M. E.; SADIK, W. A. A.; BEDIR, A. The effect of sugarcane bagasse fiber on the properties of recycled high density polyethylene, Journal of Composite Materials, v. 49, p. 3251-3262, 2015.

JESUS, Luiz Carlos Correia de. Obtenção e caracterização de compósitos de poliestireno expandido pós-consumo reforçados com celulose de bagaço de cana-de-açúcar. 2014. Dissertação (Mestrado em Integridade de Materiais da Engenharia) - Faculdade de Tecnologia, Universidade de Brasília, Brasília, 2014.

KAYMAKCI, A.; AYRILMIS, N.; AKKILIC, H. Utilization of tinder fungus as filler in production of hdpe/wood composite. Wood Research. v. 61, p. 885-894, 2016.

KABIR, M. M.; WANG, H.; LAU, K. T.; CARDONA, F. Effects of chemical treatments on hemp fibre structure. Applied Surface Science, v. 276, p. 13-23, 2013.

KU, H.; WANG, H.; PATTARACHAIYAKOOP, N.; TRADA, M. A review on the tensile properties of natural fiber reinforced polymer composites. Composites Part B: Engineering, v. 42, p. 856-873, 2011.

LEÃO, Ariadne Gonçalves de. Compósitos sustentáveis à base de polipropileno pós- consumo e fibras de bananeira modificadas por tratamento químico. 2013. Trabalho de Conclusão de Curso (Graduação em Tecnologia em Polímeros) - Escola de Engenharias, Centro Universitário Estadual da Zona Oeste, Rio de Janeiro, 2013.

LÍBANO, E. V. D. G.; GOMES, R. F. F.; MATTOS, M. D. Síntese de argila organofílica e sua incorporação via extrusão para a obtenção de compósitos com o polietileno de alta densidade. Revista Iberoamericana de Polímeros, v. 20, p. 156-167, 2019.

LIGOWSKI, E.; SANTOS, B. C.; FUJIWARA, S. T. Materiais compósitos a base de fibras da cana-de-açúcar e polímeros reciclados obtidos através da técnica de extrusão. Polímeros: Ciência e Tecnologia, v. 25, p. $70-75,2015$.

MOKHOTHU, T.H. \& JOHN, M.J. Review on hygroscopic aging of celulose fibres and their biocomposites. Carbohydrate Polymers, v. 131, p. $337-354,2015$.

MOTAUNG, T.; MOCHANE, M.; MAKHETHA, T.; MOTLOUNG, S.; MOKHOTHU, T.; MOKHENA, T.; MOJI, RANTOOA, Effect of mechanical treatment on morphology and thermal and mechanical properties of sugar cane bagasse-low-density polyethylene composites, Polymer Composites, v. 38, p. 1497-1503, 2017.

MULINARI, D. R.; VOORWALD, H. J. C; CIOFFI, M. O.; SILVA, M. L. C. P. Cellulose fiber-reinforced highdensity polyethylene composites-Mechanical and thermal properties, Journal of Composite Materials, v. 51, p. $1807-1815,2017$. 
NETO, A. G. V. C.; GANZERLI, T. A.; CARDOZO, A. L.; FÁVARO, S. L.; PEREIRA, A. G. B.; GIROTTO, E. M.; RADOVANOVIC, E. Development of composites based on recycled polyethylene/sugarcane bagasse fibers, Polymer Composites, v. 35, p. 768-774, 2014.

OLIVEIRA, Odivan Coutinho de. Avaliação de fibras de bagaço de cana-de-açúcar in natura e modificada para aplicação em compósitos. 2018. Dissertação (Mestrado em Engenharia de Ciência dos Materiais) - Centro de Ciência e Tecnologia, Universidade Estadual do Norte Fluminense Darcy Ribeiro, Campos dos Goytacazes, 2018.

PRACELLA, M.; CHIONNA, D.; ANGUILLESI, I.; KULINSKI, Z.; PIORKOWSKA, E. Functionalization, compatibilization and properties of polypropylene composites. with Hemp fibers. Composites Science and Technology, v. 66, p. 2218-2230, 2006.

SANJAY, M. R.; MADHU, P.; JAWAID, M.; SENTHAMARAIKANNAN, P.; SENTHIL, S.; PRADEEP, S. Characterization and properties of natural fiber polymer composites: A comprehensive review, Journal of Cleaner Production, v. 172, p. 566-581, 2018.

SANTOS, Evelise Fonseca. Efeito de agentes de acoplamento em compósitos de polipropileno com fibras de coco. Dissertação (Mestrado em Química) - Universidade Federal do Rio Grande do Sul - Instituto de Química, Rio Grande do Sul, 2007.

SPINACÉ, M. A. S.; LAMBERT, C. S.; FERMOSELLI, K. K. G.; PAOLI, M.A; Characterization of lignocellulosic curaua fibres, Carbohydrate Polymer, v. 77, p. 47-53, 2009.

TANOBE, V. O. A. Caracterização de fibras de esponjas de luffa cylindrica para utilização em compósitos com matriz polimérica. 2003. Dissertação (Mestrado em Engenharia de Materiais e Processos) - Setor de Tecnologia, Universidade Federal do Paraná, 2003.

TEODORO, K.B.R.; TEIXEIRA, E.M.; CORRÊA, A.C.; CAMPOS, A.; MARCONCINI, J.M.; MATTOSO, L.H.C. Whiskers de fibra de sisal obtidos sob diferentes condições de hidrólise ácida: efeito do tempo e da temperatura de extração. Polímeros, São Carlos, v. 21, p. 280-285, 2011.

VÄISÄNEN, T.; HAAPALA, A.; LAPPALAINEN, R.; TOMPPO, L. Utilization of agricultural and forest industry waste and residues in natural fiber-polymer composites: A review, Waste Management, v. 54, p. 62-73, 2016.

VÄISÄNEN, T.; DAS, OISIK; TOMPPO, LAURA. A review on new bio-based constituents for natural fiberpolymer composites, Journal of Cleaner Production, v. 149, p. 582-596, 2017. 